\title{
Numerical research of biomechanical system with SMA prosthesis
}

\author{
Andrzej Mitura ${ }^{1, *}$, Rafal Rusinek ${ }^{1}$ \\ ${ }^{1}$ Lublin University of Technology, Department of Applied Mechanics, Nadbystrzycka 36, 20-618 \\ Lublin, Poland
}

\begin{abstract}
In this paper the modelling of special biomechanical mechanism and application of shape memory materials are presented. The model of the human middle ear is made as multibody system. The basic 3 dof ear model of the healthy middle ear is modified to represent the damaged ear. A damaged of the ossicular chainis taken into account by adding gap in visco-elastic joints. In addition, an attempt of the ossicles chain reconstruction through prosthesis made of shape memory alloy is presented. Moreover, a new description of the hysteresis sub-loop which characterise prosthesis material is proposed. Finally, dynamic responses of healthy, damaged and reconstructed models of the middle ear are compared by quality index.
\end{abstract}

Keywords: multibody model, biomechanical system, shape memory alloy

\section{Introduction}

The human body is a complex object that receives information from the environment using the senses: eyesight, hearing, touch. With the development of anatomy, it was possible to know the structure of the hearing system. However, research on a living object is difficult. Therefore, the doctors and researchers strive to create models, which allow to study how a damaged or undamaged human ear works. In the literature different type of ear models are proposed. In some papers the finite element method is applied to model middle ear $[1,2]$. Substitutes models, for example the lumped parametric models are created as a alternative method. In paper [3] Feng and Gan present the multibody model, where six lumped masses are connected by visco-elastic joints. This biomechanical system includes such elements as: the external ear canal terminated with tympanic membrane, three ossicles (so called: the malleus, the incus, the stapes) and the cochlea. In paper [4] the authors limited the model only to the middle ear part. This approach allows testing the transmission of the signal (sound) through the hearing ossicles.

In the first part of this work, a three degrees of freedom model of middle ear is presented. The model is tested for two cases, the healthy and damaged. The damage of the middle ear can be caused by a past illness, sudden injury and so on. As a consequence a loss of continuity of the connection between the ossicles can cause. The separation of the middle ear bone in the model is made by adding a gap in the links between: malleus-incus

\footnotetext{
* Corresponding author: a.mitura@pollub.pl

Reviewers: Ivan Kuric, Alžbeta Sapietová
} 
or incus-stapes. The damage of this type can be cured through the middle ear prosthesis. A typical prosthesis is made of titanium and has to be cut during the operation in order to get the proper length [5]. Therefore, new material to active control length is searched. Rusinek and others propose the prostheses made of alloy with shape memory effect (in short SMA) [6], for example NITINOL. Generally, SMA is the material where the shape depends on temperature and/ or stress. In the second part of this paper a model of SMA spring used to reconstruct ossicles chain as the prosthesis is presented. The prosthesis is mounted between the tympanic membrane and the stapes. After reconstruction the model of middle ear was reduced to system with two degrees of freedom because usually the incus is removed. The dynamic responses of the considered models: the healthy ear, the damaged ear and the reconstructed ear with the help of SMA prosthesis are compared by a proposed quality index.

\section{Modelling and analysis of dynamics of the biomechanical system}

\subsection{Dynamics of healthy middle ear model}

In this section the model of the human middle ear is presented. The applied multibody system is based on proposition presented in papers $[4,6]$. Three rigid body represent ossicular bones. The symbols $m_{M}, m_{l}, m_{S}$ describe lumped masses of the malleus, the incus and the stapes, respectively. These ossicles are connected to each other and to the other elements of the human ear by visco-elastic joints (figure 1).



Fig. 1. Three dof model healthy middle ear

The values of system parameters have been taken from literature $[4,6]$ as follows : $m_{M}=25 \mathrm{mg}, m_{I}=28 \mathrm{mg}, m_{S}=1.78 \mathrm{mg}, k_{M}=1.1 \mathrm{~N} / \mathrm{mm}, k_{M I}=1000 \mathrm{~N} / \mathrm{mm}, k_{I}=0.4 \mathrm{~N} / \mathrm{mm}, k_{I S}=1.2$ $\mathrm{N} / \mathrm{mm}, c_{M}=0.06 \mathrm{Ns} / \mathrm{m}, c_{M I}=0.359 \mathrm{Ns} / \mathrm{m}, c_{I}=0.055 \mathrm{Ns} / \mathrm{m}, c_{I S}=0.0079 \mathrm{Ns} / \mathrm{m}, c_{S}=0.00174$ $\mathrm{Ns} / \mathrm{m}$. Nonlinear spring $\left(\mathrm{k}_{\mathrm{s}}\right)$ is described by a polynomial function:

$$
F_{\mathrm{s}}\left(x_{S}\right)=k_{S 0} x_{S}+k_{S 1} x_{S}^{2}+k_{S 2} x_{S}^{3}
$$

where: $k_{S 0}=0.825 \mathrm{~N} / \mathrm{mm}, k_{S I}=300 \mathrm{~N} / \mathrm{mm}^{2}, k_{S 2}=2.25 \times 10^{15} \mathrm{~N} / \mathrm{mm}^{3}$. Finally, the healthy middle ear system can be described by the following ordinary differential equations of motion:

$$
\begin{gathered}
m_{M} \ddot{x}_{M}+k_{M} x_{M}+c_{M} \dot{x}_{M}+k_{M I}\left(x_{M}-x_{I}\right)+c_{M I}\left(\dot{x}_{M}-\dot{x}_{I}\right)=q \sin \omega t \\
m_{I} \ddot{x}_{I}+k_{I} x_{I}+c_{I} \dot{x}_{I}-k_{M I}\left(x_{M}-x_{I}\right)-c_{M I}\left(\dot{x}_{M}-\dot{x}_{I}\right)+k_{I S}\left(x_{I}-x_{S}\right)+c_{I S}\left(\dot{x}_{I}-\dot{x}_{S}\right)=0
\end{gathered}
$$




$$
m_{S} \ddot{x}_{S}+k_{S 0} x_{S}+k_{S 1} x_{S}^{2}+k_{S 2} x_{S}^{3}+c_{S} \dot{x}_{S}-k_{I S}\left(x_{I}-x_{S}\right)-c_{I S}\left(\dot{x}_{I}-\dot{x}_{S}\right)=0
$$

The numerical simulations of the system described by Eqs.2-4 are made in Matlab software. Figure 2 shows the root mean square of stapes responses $R M S\left(x_{S}\right)$. The $R M S$ values is calculated in time window from 1 to 2 second. The healthy system is excited by periodic signal, where amplitude $q$ is changed from $0.1 \mathrm{~N}$ to $4 \mathrm{~N}$ and $\omega$ from 300 to 5000 $\mathrm{Hz}$.

a)

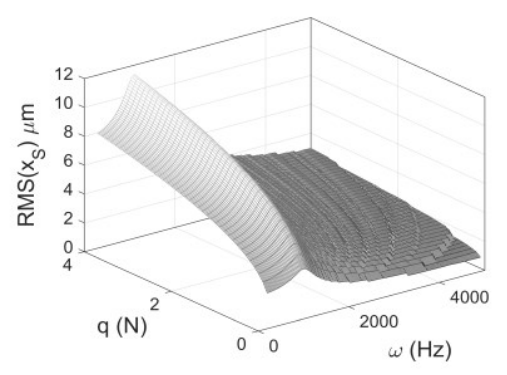

b)

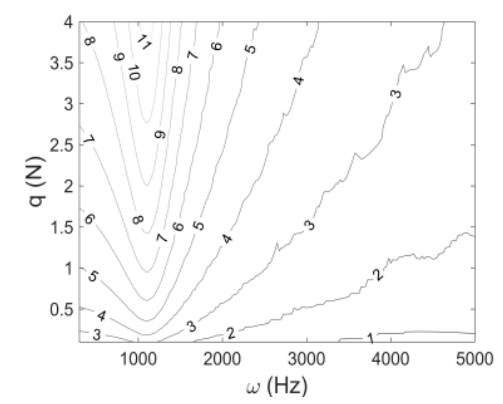

Fig. 2. The 3D characteristic of stapes responses for healthy ear (a) and its isolines interpretation (b)

In the next analyses, the characteristic presented in fig. 2 is used as reference to compare the healthy system with the damaged and reconstructed by SMA prosthesis one.

\subsection{Analysis of damaged middle ear models}

To model the damaged middle ear the model of healthy ear (fig.1) is modified. The added modifications of the model should show how loss of connection continuity in ossicles chain influences to level of stapes vibrations. In the numerical model of the damaged system the discontinuities in two connections have been assumed. Generally, the existence of the gap in visco-elastic joint can be modelled by changing the values of the stiffnes and damping coefficients to zero with no contact [7]. Two versions of the model with defect are

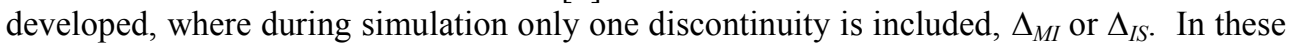
models the description of a damage has the form:

- first variant - only gap ( $\Delta_{M I}$ ) beetwen malleus and incus, then the coefficients $k_{M I}$ and $c_{M I}$ are

$$
\begin{aligned}
& k_{M I}=\left\{\begin{array}{lll}
0 & & x_{M}-x_{I} \leq \Delta_{M I} \\
1000 N / m m & \text { when } & x_{M}-x_{I}>\Delta_{M I}
\end{array}\right. \\
& c_{M I}=\left\{\begin{array}{lll}
0 & & x_{M}-x_{I} \leq \Delta_{M I} \\
0.359 N s / m & \text { when } & x_{M}-x_{I}>\Delta_{M I}
\end{array}\right.
\end{aligned}
$$

- second variant - only gap $\left(\Delta_{I S}\right)$ beetwen incus and stapes, then the coefficients $k_{I S}$ and $c_{I S}$ have form

$$
k_{I S}=\left\{\begin{array}{lll}
0 & x_{I}-x_{S} \leq \Delta_{I S} \\
1.2 N / m m & \text { when } & x_{I}-x_{S}>\Delta_{I S}
\end{array}\right.
$$




$$
c_{I S}=\left\{\begin{array}{lll}
0 & & x_{I}-x_{S} \leq \Delta_{I S} \\
0.0079 N s / m & \text { when } & x_{I}-x_{S}>\Delta_{I S}
\end{array}\right.
$$

Still the numerical simulations is made in Matlab software, where $\Delta_{M I}=0.001 \mu \mathrm{m}$ or $\Delta_{I S}=0.001 \mu \mathrm{m}$ is applied. The obtained results for the healthy system and the damaged ones (the first and the second variant) are compared using the quality index. In this paper the proposed index $J_{l}$ is defined as:

$$
J_{I}=R M S\left(x_{S}-x_{S, \text { mean }}\right)_{\text {damaged }} / R M S\left(x_{S}-x_{S, \text { mean }}\right)_{\text {healthy }}
$$

To calculate the value of this index the responses of the stapes are recorded. The root mean square is calculated for the healthy ear signal and the damaged ear system. For the damaged system the stapes responses may be significantly shifted $\left(\mathrm{x}_{\mathrm{S} \text {, mean }}\right.$ is not zero). This fact is reflected in the quality index, from displacements $x_{S}$ the mean value $x_{S \text {, mean }}$ is subtracted. Generally, index $J_{l}$ describes dynamic change of the stapes response. The stapes is last element of ossicles chain. Level of its vibrations has decisive influence to transmision signal to the internal ear.

a)

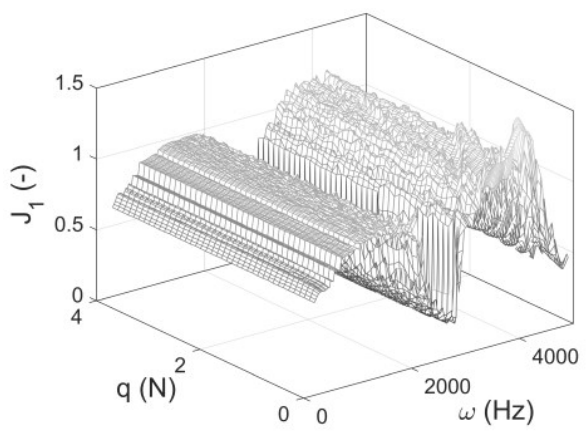

b)

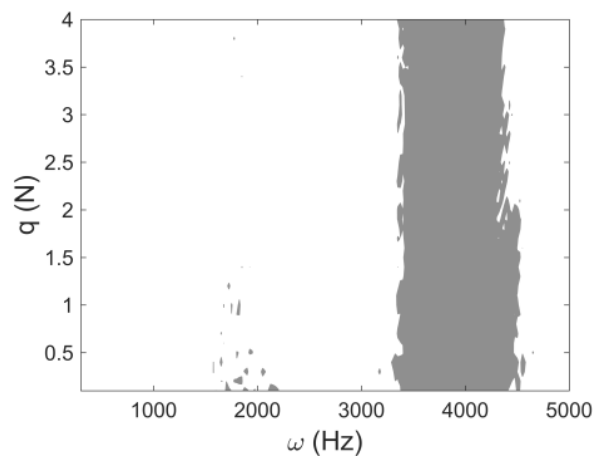

Fig. 3. The stapes responses for the damaged ear. Defect between malleus and incus, $\Delta_{M I}=0.001 \mu \mathrm{m}$. $3 \mathrm{D}$ characteristic (a) and its colormap interpretation (b), white colour $-\mathrm{J}_{1}<0$, gray colour $-\mathrm{J}_{1}>1$

a)

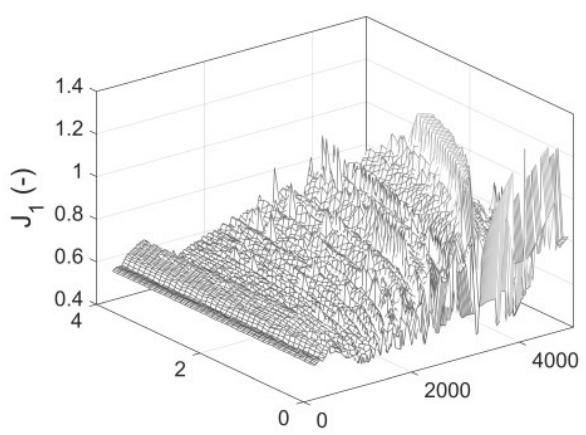

b)



Fig. 4. The stapes responses for damaged ear. Defect between incus and stapes, $\Delta_{I S}=0.001 \mu \mathrm{m}$. 3D characteristic (a) and its colormap interpretation (b), white color - $J_{I}<0$, gray color - $J_{I}>1$

The obtained results (Fig 3 and 4) present the influence of defects to vibrations of stapes. Very small gap causes a decrease in the vibration level of the last element of the 
middle ear. On the colormap in the resonance area about $1000 \mathrm{~Hz}$ dominates the white color. In damaged systems the vibrations were higher for higher frequencies (gray color, performance index $>1$ ).

\section{Model of SMA system}

\subsection{Model of the SMA prosthesis}

Damaged systems are reconstructed using SMA prosthesis. In this section the prosthesis is modeled as the spring made of shape memory alloy. It was assumed that prosthesis is a cylindrical rod with diameter $0.5 \mathrm{~mm}$ and length $4 \mathrm{~mm}$. On the basic of the paper [8] the model included pseudoelastic and hysteresis effects is developed. The idealized curve of the external hysteresis loop based on points $\left(\Delta \mathrm{x}_{1}, \mathrm{~F}_{1}\right),\left(\Delta \mathrm{x}_{2}, \mathrm{~F}_{2}\right),\left(\Delta \mathrm{x}_{3}, \mathrm{~F}_{3}\right),\left(\Delta \mathrm{x}_{4}, \mathrm{~F}_{4}\right)$ is presented in Fig.5. These points are calculated with stress and strain assumption: $\epsilon_{1}=1 \%, \epsilon_{3}=7 \%$, $\sigma_{1}=20 \mathrm{MPa}, \sigma_{3}=30 \mathrm{MPa}$. From these coordinates the cooeficients of characteristic lines are defined:

$$
\begin{array}{ll}
a_{1}=\left(F_{2}-F_{1}\right) /\left(\Delta x_{2}-\Delta x_{1}\right) & b_{1}=F_{1}-a_{1} \Delta x_{1} \\
a_{2}=\left(F_{3}-F_{4}\right) /\left(\Delta x_{3}-\Delta x_{4}\right) & b_{2}=F_{3}-a_{2} \Delta x_{3} \\
a_{0}=\left(F_{4}-F_{2}\right) /\left(\Delta x_{4}-\Delta x_{2}\right) & b_{0}=F_{2}-a_{0} \Delta x_{2}
\end{array}
$$



Fig. 5. Accepted characteristic of external loop

Here, a new model of sub-loops hysteresis is proposed. It does not describe the course of the process, phase deformation from martensite to austenite and vice versa. The model can only approximates the idealized shape of the curves. Nonlinearities are caused by stress, this is so-called pseudoelastic effect. Generated force by SMA element is described by

$$
F=b+a \Delta x
$$

Hovewer, the cooeficient $a$ and $b$ are not constant. Their values can be reinitialized. The change of coofiecitients $a(t)$ and $b(t)$ depends on following conditions:

1) if $\Delta \dot{x} \geq 0$ and $\mathrm{F} \leq \mathrm{a}_{0} \Delta \mathrm{x}+\mathrm{b}_{0}$ then $a=a_{1}, b=F-a_{1} \Delta x$,

2) if $\Delta \dot{x}<0$ and $\mathrm{F} \leq \mathrm{a}_{0} \Delta \mathrm{x}+\mathrm{b}_{0}$ then $a=0, b=F$,

3) if $\Delta \dot{x} \geq 0$ and $\mathrm{F}>\mathrm{a}_{0} \Delta \mathrm{x}+\mathrm{b}_{0}$ then $a=0, b=F$, 
4) if $\Delta \dot{x}<0$ and $\mathrm{F}>\mathrm{a}_{0} \Delta \mathrm{x}+\mathrm{b}_{0}$ then $a=a_{2}, b=F-a_{2} \Delta x$,

5) if $F \geq F_{\text {min }}$ and $\mathrm{F} \leq \mathrm{a}_{2} \Delta \mathrm{x}+\mathrm{b}_{2}$ then $a=a_{2}, b=b_{2}$,

6) if $F \leq F_{\max }$ and $\mathrm{F} \geq \mathrm{a}_{1} \Delta \mathrm{x}+\mathrm{b}_{1}$ then $a=a_{1}, b=b_{1}$.

In the proposed description of the hysteresis effect, the indicated order of checking the above conditions is important. Figure 6 and 7 present numerical results, the hysteresis loops for different load path. Coordinates of characteristics points are taken as follows: $\Delta \mathrm{x}_{1}=40 \mu \mathrm{m}, \mathrm{F}_{1}=3.92 \mathrm{~N}, \Delta \mathrm{x}_{2}=60 \mu \mathrm{m}, \mathrm{F}_{2}=5.89 \mathrm{~N}, \Delta \mathrm{x}_{3}=280 \mu \mathrm{m}, \mathrm{F}_{3}=5.89 \mathrm{~N}, \Delta \mathrm{x}_{4}=260 \mu \mathrm{m}$, $\mathrm{F}_{4}=3.92 \mathrm{~N}$.

a)



b)

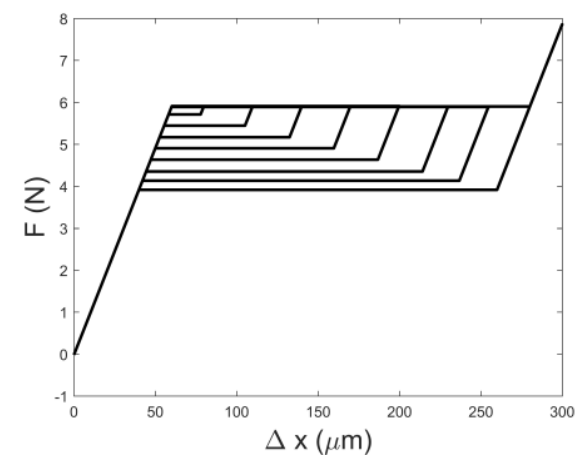

Fig. 6. First case of load path. Time series of spring deflection (a) and internal hysteresis loops (b)

a)

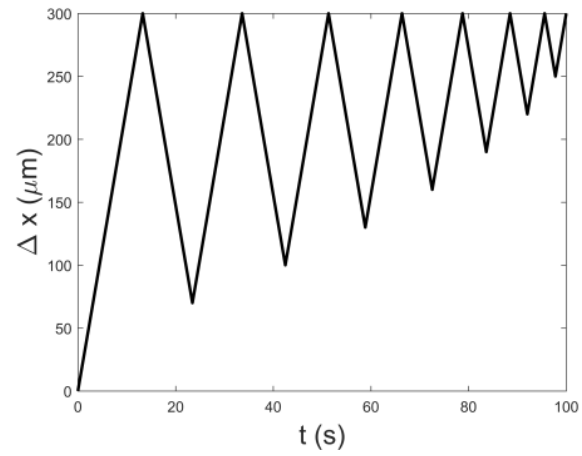

b)

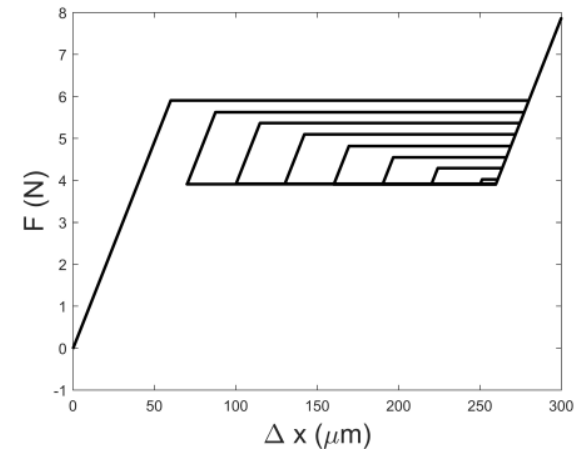

Fig. 7. Second case of load path. Time series of spring deflection (a) and internal hysteresis loops (b)

The obtained characteristics give possibility to realize the trends observed in the literature $[8,9]$.

\subsection{Ear model with SMA prosthesis}

The SMA prosthesis is usually mounted between tympanic membrane and stapes. Therefore, 2dof model of the reconstructed middle ear is proposed (figure 8). In this case the malleus and the incus are removed. The damage ossicular chain is reconstructed with prosthesis. In the proposed model the body $m_{p}$ is used to replace substitute prosthesis mass. It calculated directly from the volume of the cylindrical rod is about $5 \mathrm{mg}$ (density 6500 $\mathrm{kg} / \mathrm{m}^{3}[6]$ ). The prosthesis is held in the prescribed position through the preload $F_{\text {preload. }}$. The cylindrical rod made of SMA is squeezed by the surgeon between the membrane and the 
stapes. In the mathematical description of the reconstructed middle ear the following form of force $F$ is included:

$$
F=b+a\left(\Delta x+x_{\text {preload }}\right)-F_{\text {preload }}
$$

where $\Delta x=x_{P^{-}} x_{S}$ and $x_{\text {preload }}$ is initial prosthesis deformation generated by preload $\left(F_{\text {preload }}=2.5 \mathrm{~N}\right)$. The total force and deflection of SMA spring are defined as $\mathrm{F}_{\mathrm{SMA}}=F+F_{\text {preload }}, \Delta_{\mathrm{SMA}}=\Delta x+x_{\text {preload }}$, respectively. Modified hysteresis description form, the equation (14) based on (13) and it allows to easy consideration of the preload in the numerical model of SMA spring. Finally, 2dof system is described by equations:

$$
\begin{gathered}
m_{P} \ddot{x}_{P}+k_{M} x_{M}+c_{M} \dot{x}_{M}+F=q \sin \omega t \\
m_{S} \ddot{x}_{S}+k_{S 0} x_{S}+k_{S 1} x_{S}^{2}+k_{S 2} x_{S}^{3}+c_{S} \dot{x}_{S}-F=0
\end{gathered}
$$

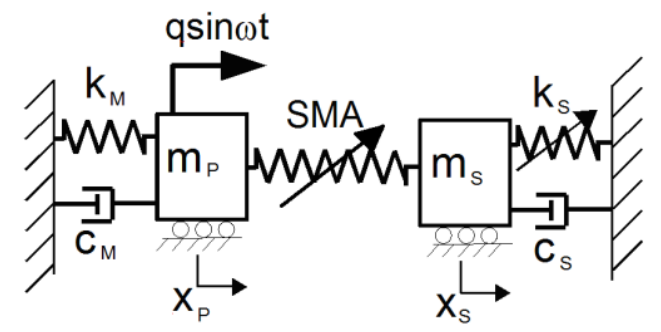

Fig. 8. Model of reconstructed middle ear with SMA prosthesis

Results of reconstructed model simulations are compared with responses of the healthy ear. A similar quality index is used as in previous section:

$$
J_{I}=R M S\left(x_{S^{-}} x_{S, \text { mean }}\right)_{\text {reconstructed }} / R M S\left(x_{S^{-}} x_{S, \text { mean }}\right)_{\text {healthy }}
$$

a)

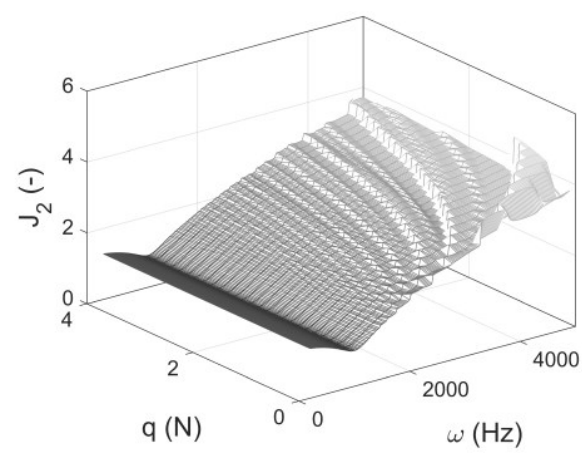

b)

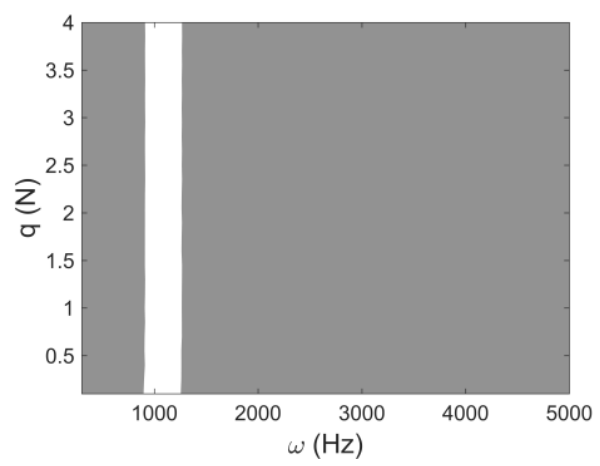

Fig. 9. The stapes responses for reconstructed model. 3D characteristic (a) and its colormap interpretation (b), white color $-\mathrm{J}_{2}<0$, gray color $-\mathrm{J}_{2}>1$

The obtained results show that proposed prosthesis can be used to reconstruction damaged ear. In this case for the majority of parameters the stapes vibrations are higher than in healthy system. Only near the resonance area are they smaller, white color on colormap. Minimal values of quality index $J_{2}$ is about 0.9 . 3D characteristics suggests that first natural frequency of reconstructed model is shifted relative to healthy ear. It can cause the problems with normal hearing at higher frequency. The detailed impact of the prosthesis parameters on the reconstructed model dynamics will be presented in the next study. The 
last stage of the research, possibility of simulating the hysteresis effect by proposed model is confirmed. Figure 10, 11 and 12 show that for larger amplitudes of excitation the hysteresis can occur. Then the stress in the SMA spring must exceed assumed $30 \mathrm{MPa}$ (or $\mathrm{F}_{\mathrm{SMA}}>5.89 \mathrm{~N}$, see section 3.1). For $\mathrm{q}=3.55 \mathrm{~N}$ internal sub-loops and for $\mathrm{q}=4 \mathrm{~N}$ only external loop can be observed.

a)



b)

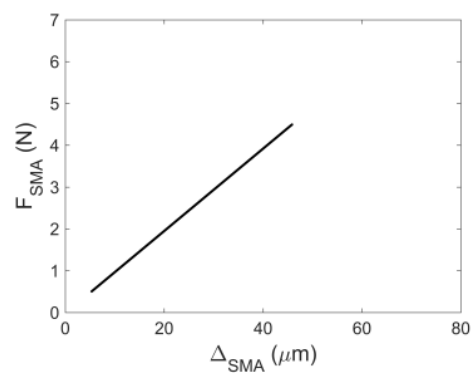

Fig. 10. Time series of stapes responses (a) and characteristic $F\left(\Delta_{S M A}\right)$ (b) for reconstructed model. $\mathrm{q}=2 \mathrm{~N}, \omega=1000 \mathrm{~Hz}$

a)



b)

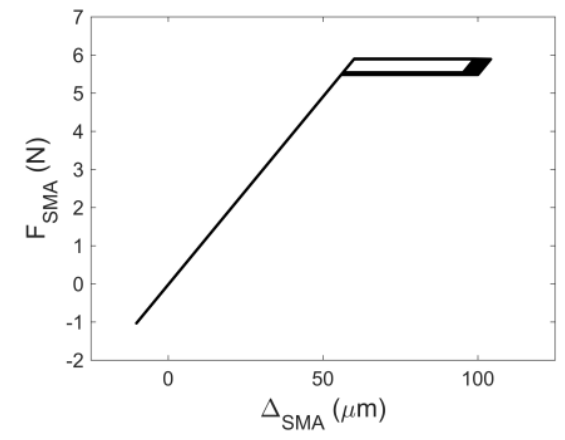

Fig. 11. Time series of stapes responses (a) and characteristic $F\left(\Delta_{S M A}\right)$ (b) for reconstructed model. $\mathrm{q}=3.55 \mathrm{~N}, \omega=1000 \mathrm{~Hz}$

a)



b)

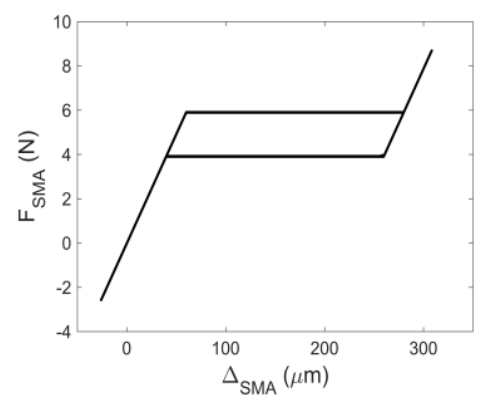

Fig. 12. Time series of stapes responses (a) and characteristic $F\left(\Delta_{S M A}\right)$ (b) for reconstructed model. $\mathrm{q}=4 \mathrm{~N}, \omega=1000 \mathrm{~Hz}$ 


\section{Conclusions}

In the paper the classic models with multiple degrees of freedom ( 2 or 3 dof $)$ have been used in modelling the human middle ear. In comparison with previous studies two discontinuities (nonlinearities) have been introduced in the ear models. First, the discontinuity of connections between the malleus and the incus or the incus and the stapes has been tested. The numerical results for very small gap (only $0.001 \mu \mathrm{m}$ ) are shown to represent ossicular chain damage. This damage causes visible decrease in stapes vibrations. From comparison both defects (gray areas in fig. 3 and 4) it can be seen that the region of undesirable signal is more increased by damage between the incus and the stapes. The reconstruction of the ossicles chain by means of SMA prosthesis gives satisfying results. This analysis will be extended in next contribution. Moreover, new model of nonlinear hysteresis effect in SMA materials is proposed. The performed simulations confirmed that proposed description is adequate to include hysteresis effect in the model of reconstructed middle ear. In the analyzed model the hysteresis effect is evident at high amplitudes of excitation. Then it is possible to obtain different hysteresis path (internal sub-loops or only external curve). In future research on reconstructed middle ear with the help of shape memory prosthesis will be developed theoretically and experimentally.

The work is financially supported under National Science Centre (Poland). Project number 2014/13/B/ST8/04047.

\section{References}

1. S.I. Chen, M.H. Lee, C.M. Yao, P.R. Chen, Y.F. Chou, T.C. Liu, Y.L. Song, C.F. Lee, Modeling sound transmission of human middle ear and its clinical applications using finite element analysis. Kaohsiung Journal of Medical Sciences 29, 133-139 (2013)

2. E. Skrodzka, J. Modlawska, Modal analysis of the human tympanic membrane of middle ear using the finite element method. Archives of Acoustics 31, 23-28 (2006)

3. B. Feng, R.Z. Gan, Lumped parametric model of the human ear for sound transmission. Biomechan Model Mechanobiol 3, 33-47 (2004)

4. R. Rusinek, J. Warminski, M. Zadrozniak, M. Szymanski, Nonlinear Approach to Modelling of Otosclerosis in a Human Middle Ear. Differ Equ Dyn Syst. 21, 45-57 (2012)

5. A. Mardassi, A. Deveze, M. Sajuan, J. Mancini, B. Parikh, A. Elbedeiwy, J. Magnan, J.P. Lavieille, Titanium ossicular chain replacement prostheses: Prognostic factors and preliminary functional results. European Annals of Otorhinolaryngology, Head and Neck Diseases 128, 53-58 (2011)

6. R. Rusinek, J. Warminski, M. Szymanski, K. Kecik, K. Kozik, Dynamics of the middle ear ossicles with an SMA prosthesis. International Journal of Mechanical Sciences 127, 163-175 (2017)

7. K.P. Jankowski, A. Mitura, J. Warminski, Dynamics of a self-balancing doublependulum system. Journal of Computational and Nonlinear Dynamics 11, 1-9 (2016)

8. Y. Huo, I. Muller, Nonequilibrium thermodynamics of pseudoelasticity. Continuum Mech Thermodyn 5, 163-204 (1993)

9. A. Masud, M. Panahandeh, F. Aurrichio, A finite-strain finite element model for the pseudoelastic behavior of shape memory alloys. Comput Methods Appl Mech Engrg. 148, 23-27 (1997) 